\title{
A Preferential Model Semantics for Default Logic
}

\author{
Frans Voorbraak
}

Logic Group

Preprint Series

No. 66

July 1991
Department of Philosophy University of Utrecht Heidelberglaan 8 3584 CS Utrecht The Netherlands 


\title{
A PREFERENTIAL MODEL SEMANTICS FOR DEFAULT LOGIC
}

\author{
Frans Voorbraak \\ Department of Philosophy, University of Utrecht \\ P.O. Box 80.126, 3508 TC Utrecht
}

\begin{abstract}
Shoham proposed a uniform approach to systems for nonmonotonic reasoning, which consists in considering standard logics augmented with a preference relation on the interpretations. Circumscription can easily seen to be a special case of this preference logic framework, but capturing default logic turned out to be more difficult, and is even thought to be impossible by some researchers of nonmonotonic reasoning. In this paper a preferential model semantics for default logic is given, by defining a preference relation on partial models called hypervaluations. Alternatively, Kripke models for $\mathbf{K} 45$ could have been used instead of hypervaluations. The given semantics slightly strengthens Shoham's notion of preferential entailment, and reflection on the question why such a strengthening is necessary provides some insight into the relation between default logic and circumscription.
\end{abstract}

1980 Mathematical Subject Classification: B22, B50

1987 Computing Reviews Classification: I.2.3

Key words and phrases: nonmonotonic resoning, default logic, preference ordering, partial interpretation.

July, 1991

To appear in the proceedings of the European Conference on Symbolic and Quantitative Approaches for Uncertainty (Marseille, October 15-17, 1991), Lecture Notes in Computer Science (Springer). 


\section{Hypervaluations}

$\mathrm{L}_{\mathrm{PL}}$ is a propositional language built on a set of propositional letters PL and the logical connectives $\neg$ for negation and $\vee$ for disjunction. $\wedge, \supset, \equiv$ are assumed to be defined in the usual way.

\section{DEFINITION 2.1}

(i) An hyperinterpretation $\mathrm{I}$ is a set of 2-valued interpretations $\mathrm{J}: \mathrm{PL} \rightarrow\{\mathrm{T}, \mathrm{F}\}$.

(ii) For any 2-valued interpretation $\mathrm{J}, \mathrm{V}_{\mathrm{J}}: \mathrm{L}_{\mathrm{PL}} \rightarrow\{\mathrm{T}, \mathrm{F}\}$ denotes the usual 2-valued valuation induced by $\mathrm{J} . \mathrm{V}_{\mathrm{J}}(\varphi)=\mathrm{T}$ and $\mathrm{V}_{\mathrm{J}}(\varphi)=\mathrm{F}$ will sometimes be written as $\vDash 2, \mathrm{~J} \varphi$ and $=2, \mathrm{~J} \varphi$, respectively.

(iii) The hypervaluation $\mathrm{V}_{\mathrm{I}}: \mathrm{LPL}_{\mathrm{PL}} \rightarrow\{\mathrm{T}, \mathrm{F}, \mathrm{U}\}$ induced by the hyperinterpretation $\mathrm{I}$ is given by

$$
\begin{array}{cl}
\mathrm{V}_{\mathrm{I}}(\varphi)=\quad & \mathrm{T} \text { iff } \forall \mathrm{J} \in \mathrm{IV}_{\mathrm{J}}(\varphi)=\mathrm{T} \\
\mathrm{F} \text { iff } \forall \mathrm{J} \in \mathrm{I}_{\mathrm{J}}(\varphi)=\mathrm{F} \\
\mathrm{U} \text { otherwise. }
\end{array}
$$

$\mathrm{V}_{\mathrm{I}}(\varphi)=\mathrm{T}$ and $\mathrm{V}_{\mathrm{I}}(\varphi)=\mathrm{F}$ will sometimes be written as $\vDash_{\mathrm{h}, \mathrm{I}} \varphi$ and $=_{\mathrm{h}, \mathrm{I}} \varphi$, respectively.

The definition of hypervaluations is strongly reminiscent of the definition of van Fraassen's supervaluations. In fact, a supervaluation corresponds with a hypervaluation whose elements are the complete extensions of some 3 -valued interpretation. The consequence relation $\vDash_{\mathrm{x}}$ induced by $\vDash_{\mathrm{x}, \mathrm{I}}$ is defined by $\Gamma \vDash_{\mathrm{x}} \Delta$ iff $\forall \mathrm{I}\left(\forall \psi \in \Gamma \vDash_{\mathrm{x}, \mathrm{I}} \psi \Rightarrow \exists \varphi \in \Delta \mathrm{F}_{\mathrm{x}, \mathrm{I}} \varphi\right)$. We do not require that $\forall \mathrm{I}\left(\forall \varphi \in \Delta f_{\mathrm{X}, \mathrm{I}} \varphi \Rightarrow \exists \psi \in \Gamma f_{\mathrm{x}, \mathrm{I}} \psi\right.$ ), as some authors do (cf. Blamey (1986)). This stronger definition would for example render the inference from $\{p \vee q, \neg q\}$ to $p$ invalid. (A counterexample would be $I=\left\{J, J^{\prime}\right\}$, where $J(p)=J^{\prime}(p)=F, J(q)=T$, and $J^{\prime}(q)=F$.)

It is well-known that the supervaluation consequence relation $\models_{S}$ is strictly weaker than the ordinary consequence relation. (See e.g. Langholm (1988).) The hypervaluation consequence relation $\mathrm{h} h$ is weaker still:

\section{PROPOSITION $2.2 \quad \vDash_{\mathrm{h}} \subset \vDash_{\mathrm{S}} \subset \vDash_{2}$.}

Proof. $\left.\right|_{\mathrm{h}} \subseteq F_{\mathrm{S}} \subseteq \mid=2$ is trivial. $\left.\right|_{\mathrm{S}} \nsubseteq \mid=_{\mathrm{h}}$ follows from the fact that $\{\mathrm{p} \vee \mathrm{q}\} F_{\mathrm{S}}\{\mathrm{p}, \mathrm{q}\}$, whereas $\{\mathrm{p} \vee \mathrm{q}\} \not \not_{\mathrm{h}}\{\mathrm{p}, \mathrm{q}\} .||_{2} \nsubseteq F_{\mathrm{S}}$ follows from the fact that $\models_{2}\{\mathrm{p}, \neg \mathrm{p}\}$, whereas $\neq_{\mathrm{s}}\{\mathrm{p}, \neg \mathrm{p}\}$.

It is easy to see that $l_{h}, l_{\mathrm{S}}$, and $l_{2}$ coincide in case the consequence set $\Delta$ is $\varnothing$ or a singleton. It is also clear that $\Gamma \vDash_{h} \Delta$ iff for every non-empty hyperinterpretation $I\left(\forall \psi \in \Gamma \vDash_{h, I} \psi \Rightarrow \exists \varphi\right.$ $\left.\in \Delta l_{h, I} \varphi\right)$. Further, $l_{h}$ is closely related to the consequence relations of the modal logics K45, KD45, and S5:

PROPOSITION 2.3 Let $\Gamma, \Delta \subseteq \mathrm{LPL}_{\mathrm{PL}}$, let for any $\Omega \subseteq \mathrm{LPL}_{\mathrm{PL}} \square \Omega$ denote the set $\{\square \varphi \mid \varphi \in \Omega\}$, and let $\mathbf{S} \in\{\mathbf{K 4 5}, \mathbf{K D} 45, \mathbf{S 5}\}$. Then $\Gamma\left|{ }_{\mathrm{h}} \Delta \Leftrightarrow \square \Gamma\right|=\mathbf{S} \square \Delta$. 
Proof. It is easy to see that there exists a 1-1 correspondence $\mu$ between non-empty hyperinterpretations and distinguishable connected $\mathbf{S} 5$ models such that $\mathrm{V}_{\mathrm{I}}(\varphi)=\mathrm{T}$ iff $\mu(\mathrm{I}) \vDash \square \varphi$. The equivalence of $\models_{\mathbf{S} 5}, l_{\mathbf{K D} 45}$, and $\models_{\mathbf{K} 45}$ restricted to boxed formulas is well-known.

The above proposition justifies to some extent the epistemic reading of the hypervaluation consequence relation which was mentioned in the introduction, since $K(D) 45$ is widely regarded to be a good candidate for the logic of the beliefs of a rational agent, whereas $\mathbf{S 5}$ is often mentioned as a suitable logic of knowledge. Below we mention some properties of $l_{h}$.

Proposition $2.4 \quad$ Suppose $\Gamma \vDash_{\mathrm{h}} \Delta$ and $\Delta \neq \varnothing$. Then $\exists \varphi \in \Delta$ s.t. $\left.\Gamma\right|_{\mathrm{h}} \varphi$.

COROLlary $2.5 \quad$ Suppose $\Delta \neq \varnothing$. Then $\Gamma \vDash_{\mathrm{h}} \Delta$ iff $\exists \varphi \in \Delta$ s.t. $\Gamma \vDash_{2} \varphi$.

COROLLARY 2.6 (Compactness) If $\left.\Gamma\right|_{\mathrm{h}} \Delta$, then there exists finite sets $\Gamma^{\prime}, \Delta^{\prime}$ such that $\Gamma^{\prime}$ $\subseteq \Gamma, \Delta^{\prime} \subseteq \Delta$, and $\left.\Gamma^{\prime}\right|_{h} \Delta^{\prime}$.

COROLLARY $2.7 \quad \Gamma \models_{\mathrm{h}} \Delta$ is decidable for finite $\Gamma$ and $\Delta$.

Hypervaluations are generalized to first-order languages in the obvious way: First-order hyperinterpretations are just sets of ordinary first-order interpretations, and the first-order hypervaluation induced by a first-order hyperinterpretation I is defined as in the propositional case. Propositions 2.2 and 2.4 remain valid in the first-order case, and so do corollaries 2.5 and 2.6. For corollary 2.7 the decidability of propositional $l_{2}$ is of course essential. The first-order analogue of proposition 2.3 will only be valid for a suitable first-order generalization of the propositional Kripke models. Such a generalization is given in Voorbraak (forthcoming).

\section{Default logic and preferential model semantics}

For convenience, we repeat some basic definitions of default logic. For a proper introduction, see e.g. Reiter (1980), Etherington (1988), or Lukaszewicz (1990). Our presentation of the preferential model semantics relies heavily on Makinson (1989).

Let L be some (standard) first-order language and let Th : $\wp \mathrm{L} \rightarrow \wp \mathrm{L}$ be given by $\mathrm{Th}(\Sigma)=\{\varphi \mid$ $\left.\Sigma l_{=2} \varphi\right\} . \alpha, \beta, \ldots$ denote formulas of $\mathrm{L}$.

DEFINITION 3.1 A default rule (or simply a default) is an expression of the form $\alpha$ : $\beta_{1}, \ldots, \beta_{n} / \omega \quad(n \geq 1) . \alpha$ is called the prerequisite, $\beta_{1}, \ldots, \beta_{n}$ the justifications, and $\omega$ the consequent of the rule. A default rule $\alpha: \beta_{1}, \ldots, \beta_{n} / \omega$ is called closed iff the set of its free variables $\mathrm{FV}\left\{\alpha, \beta_{1}, \ldots, \beta_{\mathrm{n}}, \omega\right\}=\varnothing$. 
DEFINITION 3.2 A default theory is a pair $\langle\mathrm{D}, \mathrm{W}\rangle$, where $\mathrm{D}$ is a set of defaults and $\mathrm{W}$ is a set of closed formulas of L. A default theory is called closed iff every default in D is closed.

DEFINITION 3.3 $E$ is an extension of a closed default theory $\langle D, W\rangle$ iff $E=\cup_{i} \geq 0$, where

$\cdot \mathrm{E}_{0}=\mathrm{W}$

- $E_{i+1}=\operatorname{Th}\left(E_{i}\right) \cup\left\{\omega \mid \alpha: \beta_{1}, \ldots, \beta_{n} / \omega \in D, \alpha \in E_{i}\right.$ and $\left.\forall j \in\{1, ., n\} \neg \beta_{j} \notin E\right\}$.

An extension of a default theory $\langle\mathrm{D}, \mathrm{W}\rangle$ is intended to represent a reasonable state of belief based on the defaults in $\mathrm{D}$ and on the propositions in W. As extensions of a default theory $\langle\mathrm{D}, \mathrm{W}\rangle$ which is not closed one simply takes the extensions of the closed default theory $\left\langle D^{\prime}, W\right\rangle$, where, roughly speaking, D' is obtained from D by taking all closed instances of D. (Since the defaults of $D^{\prime}$ may contain Skolem constants, the extensions of $\left\langle D^{\prime}, W\right\rangle$ have to be restricted to L. Details can be found in e.g. Lukaszewicz (1990).)

DEFINITION $3.4 \quad$ Let $\delta=\alpha: \beta_{1}, . ., \beta_{\mathrm{n}} / \omega$ be a closed default and let $\Gamma$ be a set of first-order models. The preference relation corresponding to $\delta, \geq_{\delta}$, over $\wp \Gamma$ is defined as follows:

$$
\begin{aligned}
\Gamma_{1} \geq_{\delta} \Gamma_{2} \text { iff } & \forall M \in \Gamma_{2} M \mid=2 \alpha, \\
& \forall i \in\{1, \ldots, n\} \exists M_{i} \in \Gamma_{2} M_{i} \models_{2} \beta_{i}, \\
& \text { and } \Gamma_{1}=\Gamma_{2}-\{M|M| \neq 2 \omega\} .
\end{aligned}
$$

Intuitively, $\Gamma_{1} \geq_{\delta} \Gamma_{2}$ means that on account of $\delta$ the (partial) world-description $\Gamma_{1}$ is preferred to the (partial) world-description $\Gamma_{2}$. The preference relation $\geq_{D}$ corresponding to a set of defaults $\mathrm{D}$ is simply the transitive closure of the union of the preference relations corresponding to the elements of $\mathrm{D}$. Notice that, in spite of the suggestive notation, $\geq_{\delta}$ and $\geq_{\mathrm{D}}$ are not necessarily partial orderings, since in general neither of them is reflexive. Using the reflexive closures of $\geq \delta$ and $\geq_{D}$ instead of the relations themselves would have worked as well, but we stick to the definition given in Etherington (1988).

Let $\mathrm{MOD}(\mathrm{W})$ be the set of first-order models of $\mathrm{W}$. The extensions of a closed default theory $\langle\mathrm{D}, \mathrm{W}\rangle$ correspond with the $\geq_{\mathrm{D}}$-maximal elements of $\wp(\mathrm{MOD}(\mathrm{W}))$ which have some additional property called stability:

DEFINITION 3.5 Let $\Delta=\langle\mathrm{D}, \mathrm{W}\rangle$ be a closed default theory and let $\Gamma \subseteq \mathrm{MOD}(\mathrm{W})$.

$\Gamma$ is called stable for $\Delta$ iff $\exists D^{\prime} \subseteq D$ such that $\Gamma \geq D^{\prime} M O D(W)$ and for every justification $\beta$ of a default of $D^{\prime} \exists M \in \Gamma M \vDash 2 \beta$.

PROPOSITION 3.6 (Etherington (1988)) Let $\Delta=\langle\mathrm{D}, \mathrm{W}\rangle$ be a closed default theory.

(i) If $E$ is an extension of $\Delta$, then $\{M \mid M \models 2 E\}$ is stable for $\Delta$ and a $\geq_{D}$-maximal element of $\wp(\mathrm{MOD}(\mathrm{W}))$.

(ii) If $\Gamma$ is stable for $\Delta$ and $a \geq D$-maximal element of $\wp(M O D(W))$, then $\{\varphi|\forall M \in \Gamma M|=2$ $\varphi\}$ is an extension of $\Delta$. 
In the above proposition, $\geq_{D}$ can be replaced by $\geq_{\Delta}$, defined by $\Gamma_{1} \geq_{\Delta} \Gamma_{2}$ iff $\Gamma_{1} \geq_{D} \Gamma_{2} \geq_{D}$ $\operatorname{MOD}(\mathrm{W})$ or $\Gamma_{1} \geq_{\mathrm{D}} \Gamma_{2}=\mathrm{MOD}(\mathrm{W})$.

DEFINITION 3.7 A preferential model structure (p.m.s.) for $\mathrm{L}$ is a tuple $\mathrm{pr}=\langle\mathcal{M}, \mid=, \sqsubset\rangle$, where $\mathcal{M}$ is a set of models, $\vDash \subseteq \mathcal{M} \times \mathrm{L}$ is a satisfaction relation, and $\sqsubset \subseteq \mathcal{M} \times \mathcal{M}$. We often write $\mathrm{M} \vDash \varphi$ for $\langle\mathrm{M}, \varphi\rangle \in \mathrm{l}=$.

For technical reasons, Makinson (1989) imposes no constraints on $\vDash$ and $\sqsubset$. However, Shoham $(1987,1988)$ seems to allow only partial orderings $[$ and models of standard logics. He does not make precise what he understands by 'standard' and just assumes standard logics to be propositional or predicate (modal) logics, but a reasonable interpretation of his intentions is to require that $\vDash$ induces a (standard) consequence relation satisfying inclusion, idempotency, and monotony. The preferential model structures proposed below are also preferential model structures in this stricter sense.

DEFINITION $3.8 \quad$ Let $\mathrm{pr}=\langle\mathcal{M}, \mid=, \sqsubset \overline{\text { b }}\rangle$ be a p.m.s. for $\mathrm{L}$.

(i) $\quad M \in \mathcal{M}$ preferentially satisfies $\Gamma \subseteq \mathrm{L}$, notation $\mathrm{M}={ }_{\mathrm{pr}} \Gamma$, iff $\mathrm{M} \vDash \Gamma$ (i.e., $\forall \varphi \in \Gamma \mathrm{M}=\varphi$ ) and $\neg \exists \mathrm{M}^{\prime} \sqsupset \mathrm{M} \mathbf{M}^{\prime} \mid=\Gamma$. The set of preferred models $\mathcal{M}_{\mathrm{pr}}=\left\{\mathrm{M} \in \mathcal{M}|\exists \Gamma \subseteq \mathrm{L} \mathrm{M}|={ }_{\mathrm{pr}}\right.$ $\Gamma\}$. We will usually write $M \models_{\mathrm{pr}} \varphi$ instead of $\mathrm{M} \models_{\mathrm{pr}}\{\varphi\}$.

(ii) The operation $\mathrm{Th}_{\mathrm{pr}}: \wp \mathrm{L} \rightarrow \wp \mathrm{L}$ of preferential entailment is given by $\varphi \in \mathrm{Th}_{\mathrm{pr}}(\Gamma)$ iff $\forall \mathrm{M}(\mathrm{M} \vDash \mathrm{pr} \Gamma \Rightarrow \mathrm{M} \vDash \varphi)$.

(iii) $\quad \Gamma$ is called preferentially satisfiable iff $\exists \mathrm{MM} \vDash_{\mathrm{pr}} \Gamma$.

(iv) $\quad \Gamma$ is called preferentially valid iff $\Gamma \subseteq \mathrm{Th}_{\mathrm{pr}}(\varnothing)$.

Notice that $\varphi$ is preferentially valid iff $\varphi$ is true in every preferred model of $\varnothing$, or, in other words, iff for every $\sqsubset$-maximal model $M M=\varphi$. Shoham gives a slightly different, and in our opinion less intuitive, definition of preferential validity, namely $\varphi$ is preferentially valid iff $\neg \varphi$ is not preferentially satisfiable. In general, both definitions are not equivalent.

DEFINITION 3.9 Let $\Delta=\langle\mathrm{D}, \mathrm{W}\rangle$ be a closed default theory.

(i) $\quad \mathrm{m}$ is called $\Delta$-bounded iff $\exists \mathrm{m}^{\prime} \geq_{\Delta} \mathrm{m}$ such that $\mathrm{m}^{\prime}$ is $\geq_{\Delta}$-maximal and stable for $\Delta$.

(ii) The p.m.s. associated with $\Delta$ is the p.m.s. $\operatorname{pr}(\Delta)=\langle\mathcal{M}, \models, \sqsubset \bar{l}\rangle$, where $\mathcal{M}=\wp(\operatorname{MOD}(\mathrm{W}))$, $\vDash=\{\langle\mathrm{m}, \varphi\rangle \mid \mathrm{m} \in \mathcal{M}, \varphi \in \mathrm{L}$, and $\mid=\mathrm{h}, \mathrm{m} \varphi\}$, and $\sqsubset$ is defined as follows:

$\mathrm{m} \sqsupset \mathrm{m}^{\prime}$ iff $\mathrm{m}$ is $\Delta$-bounded and $\mathrm{m}^{\prime}$ is not $\Delta$-bounded, or $\mathrm{m}$ and $\mathrm{m}^{\prime}$ are not $\Delta$-bounded and $\mathrm{m}^{\prime} \neq \mathrm{m}=\varnothing$, or $\mathrm{m}$ and $\mathrm{m}^{\prime}$ are $\Delta$-bounded, $\mathrm{m} \neq \mathrm{m}^{\prime}$, and $\mathrm{m} \geq_{\Delta} \mathrm{m}^{\prime}$.

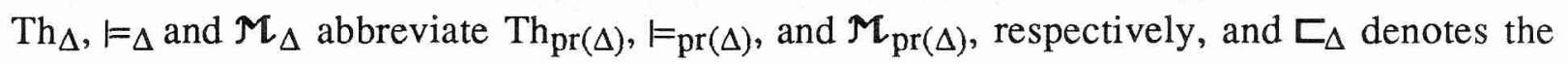
preference relation of $\operatorname{pr}(\Delta)$. $\Sigma_{\Delta}$ is a strict p.o. and $\mathcal{M}_{\Delta}=\{\varnothing\} \cup\left\{\mathrm{m} \in \mathcal{M} \mid \neg \exists \mathrm{m}^{\prime} \sqsupset_{\Delta} \mathrm{m}\right\}$. Hence the only preferred model which is not necessarily $\Sigma_{\Delta}$-maximal is the trivial model $\varnothing$. 
PROPOSITION 3.10 Let $\Delta=\langle\mathrm{D}, \mathrm{W}\rangle$ be a closed default theory and $\operatorname{pr}(\Delta)=\langle\mathcal{M}|=,, C, \quad$ its associated p.m.s.

(i) If $\mathrm{E}$ is an extension of $\Delta$, then $\left\{\mathrm{M} \mid \mathrm{M} \vDash_{2} \mathrm{E}\right\} \vDash_{\Delta} \mathrm{E}$.

(ii) If $\mathrm{m} \in \mathcal{M}_{\Delta}$ and $\mathrm{m} \neq \varnothing$, then $\{\varphi \mid \mathrm{m} \vDash \varphi\}$ is a consistent extension of $\Delta$.

(iii) If $\mathcal{M}_{\Delta}=\{\varnothing\}$, then $\Delta$ has no consistent extension.

(iv) $\mathrm{Th}_{\Delta}(\Gamma)=\cap\{\mathrm{E} \mid \mathrm{E}$ is an extension of $\Delta$ such that $\Gamma \subseteq \mathrm{E}\}$.

Proof. (i) If $\mathrm{E}$ is an extension of $\Delta$, then, by proposition $3.6, \mathrm{~m}=\{\mathrm{M} \mid \mathrm{M} \vDash 2 \mathrm{E}\}$ is $\geq \mathrm{D}-\mathrm{maximal}$ and stable for $\Delta$. Hence $\neg \exists \mathrm{m}^{\prime} \sqsupset \mathrm{m}$. Since $\mathrm{m} \vDash \mathrm{E}$, we have $\mathrm{m} \vDash=_{\Delta} \mathrm{E}$.

(ii) If $m \in \mathcal{M}_{\Delta}$ and $\mathrm{m} \neq \varnothing$, then $\mathrm{m}$ is $\geq_{\mathrm{D}}$-maximal and stable for $\Delta$. Hence, by proposition 3.6, $\{\varphi \mid \mathrm{m} \vDash \varphi\}$ is an extension of $\Delta$. Since $\mathrm{m} \neq \varnothing$, this extension is consistent.

(iii) If $\mathcal{M}_{\Delta}=\{\varnothing\}$, then either there are no $\Delta$-bounded models, and therefore no extensions for $\Delta$, or $\varnothing$ is $\Delta$-bounded, and thus $\{\varphi|\varnothing|=\varphi\}$ is the unique, inconsistent extension of $\Delta$.

(iv) Assume $\varphi \in \mathrm{Th}_{\Delta}(\Gamma)$ and let $E$ be an extension of $\Delta$ such that $\Gamma \subseteq \mathrm{E}$. Then, as in (i), $\neg \exists \mathrm{m}^{\prime}$ $\sqsupset \mathrm{m}=\left\{\mathrm{M} \mid \mathrm{M} \vDash_{2} \mathrm{E}\right\}$. Since $\mathrm{m} \vDash \Gamma$, we have $\mathrm{m} \vDash_{\Delta} \Gamma$. Hence $\mathrm{m} \vDash \varphi$, and thus $\varphi \in \mathrm{E}$.

On the other hand, assume $\varphi \notin T h_{\Delta}(\Gamma)$. Then $\exists \mathrm{m}$ such that $\mathrm{m} \vDash_{\Delta} \Gamma$ and $\mathrm{m} \mid \neq \varphi$. By (ii), $\mathrm{E}=\{\psi \mid$ $\mathrm{m} \vDash \psi\}$ is an extension of $\Delta$ and clearly $\Gamma \subseteq \mathrm{E}$. Since $\varphi \notin \mathrm{E}, \varphi \notin \cap\{\mathrm{E} \mid \mathrm{E}$ is an extension of $\Delta$ such that $\Gamma \subseteq \mathrm{E}\}$.

Notice that $\varnothing$ being the only preferred model corresponds to $\Delta$ having an inconsistent extension or having no extension at all. It is possible to give an alternative definition of $\sqsubset_{\Delta}$ such that $\varnothing \in$ $\mathcal{M}_{\Delta}$ iff $\Delta$ has an inconsistent extension and $\mathcal{M}_{\Delta}=\varnothing$ iff $\Delta$ has no extension. However, this alternative $\sqsubset_{\Delta}$ will in general not be a strict partial ordering, since for any pr $=\langle\mathcal{M}, \models, \sqsubset\rangle$ with finite $\mathcal{M}$ and s.p.o. $\sqsubset$ we have $\mathcal{M}_{\mathrm{pr}} \neq \varnothing$. The collapsing of the cases that $\Delta$ has an inconsistent extension and that $\Delta$ has no extension can be defended by pointing out that both are boundary cases added for technical convenience, rather than representations of belief states of truly rational agents. (In both cases a rational agent would have to revise his belief state.)

An immediate corollary of proposition 3.10(iv) is the monotony of $\mathrm{Th}_{\Delta}$. Hence as long as you keep the default theory constant, the reasoning is monotonic. Default consequence is nonmonotonic because default theories are updated in the light of new information. To capture this in terms of preferential model semantics, we propose a strengthening of the notion of preferential consequence and we define a preferential model semantics for a class of default theories with the same defaults. The strong notion of preferential consequence is equivalent to the usual notion in case the models are two-valued. Applied to hypervaluations, the strong notion takes account of the intuition that default extensions-the preferred models of default theory - have to be grounded on the facts. The definition of a p.m.s. associated with a class of defaults is a global version of the corresponding definition for a single default theory.

DEFINITION 3.11 Let $\mathrm{pr}=\langle\mathcal{M},=, \sqsubset \varnothing\rangle$ be a p.m.s. $\mathbf{M} \in \mathcal{M}$ strongly preferentially satisfies $\Gamma$, notation $M \mid=p r^{*} \Gamma$, iff $M \vDash{ }_{p r} \Gamma$ and $\forall M^{\prime}\left(\Gamma \subseteq\left\{\varphi \mid M^{\prime} \vDash \varphi\right\} \subset\{\varphi|M| \varphi\} \Rightarrow M^{\prime} \sqsubset M\right) . \varphi \in$ $\mathrm{Th}_{\mathrm{pr}}(\Gamma)$ iff $\forall \mathrm{M}\left(\mathrm{M} \vDash \mathrm{pr}^{*} \Gamma \Rightarrow \mathrm{M} \vDash \varphi\right)$ 
DEFINITION 3.12 Let D be a set of closed defaults. The p.m.s. associated with $D$ is the p.m.s. $\operatorname{pr}(\mathrm{D})=\langle\mathcal{M}, \vDash, C\rangle$, where $\mathcal{M}=\wp(\operatorname{MOD}(\varnothing)), \vDash=\left\{\langle\mathrm{m}, \varphi\rangle \mid \mathrm{m} \in \mathcal{M}, \varphi \in \mathrm{L}\right.$, and $\left.=_{\mathrm{h}, \mathrm{m}} \varphi\right\}$, and $ᄃ$ is defined as follows: $m \sqsupset m^{\prime}$ iff $m \beth_{\Delta} m^{\prime}$, where $\Delta=\left\langle D,\left\{\varphi \mid m^{\prime} \vDash \varphi\right\}\right\rangle$.

We write $\sqsubset_{D}$ for the preference relation of $\operatorname{pr}(D)$ and we use obvious abbreviations, such as

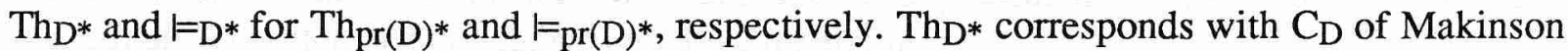
(1989), since $\mathrm{Th}_{\mathrm{D}^{*}}(\Gamma)$ is the intersection of all extensions of $\langle\mathrm{D}, \Gamma\rangle$.

PROPOSITION 3.13 $\operatorname{Th}_{\mathrm{D}} *(\Gamma)=\bigcap\{\mathrm{E} \mid \mathrm{E}$ is an extension of $\langle\mathrm{D}, \Gamma\rangle\}$.

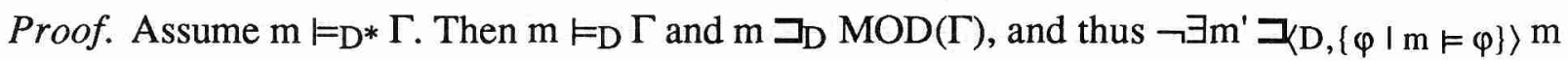

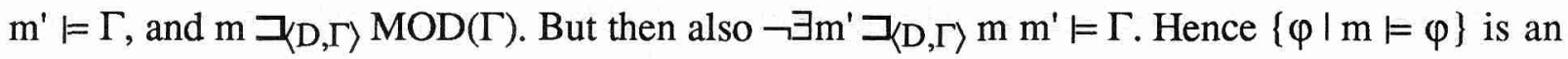
extension of $\langle\mathrm{D}, \Gamma\rangle$. On the other hand, assume that $\{\varphi \mid \mathrm{m} \vDash \varphi\}$ is an extension of $\langle\mathrm{D}, \Gamma\rangle$. Then $\mathrm{m} \vDash \Gamma$ and $\neg \exists \mathrm{m}^{\prime} \sqsupset_{\mathrm{D}} \mathrm{m} \mathrm{m}^{\prime} \vDash \Gamma$. Thus $\mathrm{m} \vDash \mathrm{D} \Gamma$. Let $\mathrm{m}^{\prime}$ be such that $\Gamma \subseteq\left\{\varphi\left|\mathrm{m}^{\prime}\right|=\varphi\right\} \subset\{\varphi \mid \mathrm{m}$ $\vDash \varphi\} \Rightarrow$ Then $\mathrm{m} \sqsupset \mathrm{D} \mathrm{m}^{\prime}$, since each default that is applied to get from $\operatorname{MOD}(\Gamma)$ to $\mathrm{m}$ can be applied to get from $m^{\prime}$ to $m$. Hence $m \vDash n^{*} \Gamma$. We can conclude that $m \vDash D^{*} \Gamma$ iff $\{\varphi \mid m \vDash \varphi\}$ is an extension of $\langle\mathrm{D}, \Gamma\rangle$, and the proposition follows immediately.

In view of proposition 2.4 it is no surprise that one can also obtain a modal preferential model semantics for default logic. There is a quite obvious reformulation of the above results in terms of K45 models instead of hypervaluations. The use of KD45 or S5 is less straightforward since these logics lack models matching inconsistent extensions. If one assumes extensions to be consistent, then, from a technical point of view, default rules can be interpreted as well in terms of (KD45-)belief as in terms of (S5-)knowledge. See Voorbraak (forthcoming).

\section{Discussion}

We have given a preferential model semantics for default consequence understood in the 'meet' or 'sceptical' sense. ( $\varphi$ is a sceptical default consequence of $\Delta$ iff $\varphi$ is true in every extension of $\Delta$.) Makinson (1989) has shown that credulous default consequence ( $\varphi$ is a credulous default consequence of $\Delta$ iff $\varphi$ is true in some (arbitrarily chosen) extension of $\Delta$ ) does not satisfy cumulative transitivity $(\Gamma \subseteq \Delta \subseteq \operatorname{Cn}(\Gamma) \Rightarrow \operatorname{Cn}(\Delta) \subseteq \operatorname{Cn}(\Gamma)$ ) and can therefore not be captured in a p.m.s. (Makinson's result that $\mathrm{Th}_{\mathrm{pr}}$ satisfies cumulative transitivity also holds for $\mathrm{Th}_{\mathrm{pr}}{ }^{*}$ ) Sceptical default consequence does satisfy cumulative transitivity, although it is still not a cumulative consequence operation, since it does not satisfy cumulative monotony ( $\Gamma \subseteq \Delta \subseteq$ $\operatorname{Cn}(\Gamma) \Rightarrow \operatorname{Cn}(\Gamma) \subseteq \operatorname{Cn}(\Delta))$

A failure of cumulative monotony implies that (implicitly) facts and derived formulas have a different status. But nonmonotonic formalisms which distinguish facts from derived formulas may very well be cumulative. The failure of cumulative monotony in default logic 
seems to be a corollary of the requirement that extensions have to be grounded on the facts. Learning new facts, even previously derivable ones, can result in more grounded (partial) world descriptions. This might be defended by taking the difference between facts and derived formulas serious. Alternatively, one could argue that one should not require that the logic is cumulative, but rather that the state of belief of an ideally rational agent should not contain a set of defaults $\mathrm{D}$ such that $\mathrm{Th}_{\mathrm{D}}$ * fails cumulative monotony.

In a forthcoming paper (Voorbraak (forthcoming)) we argue that both default and superstrongly autoepistemic extensions can be obtained by applying essentially only two different filters, which can roughly be described as taking justification-minimal models and taking grounded models, respectively. A model is called justification-minimal iff the set of false justifications is minimal. Justification minimization is implemented in default logic by requiring the $\geq_{D}$-maximal elements to be stable, it is implemented in autoepistemic logic by strengthening minimal $\mathrm{AE}$ extensions to superstrongly grounded $\mathrm{AE}$ extensions, and it is closely related to the minimization in circumscription.

The groundedness filter requires the extensions of a default theory $\langle D, W\rangle$ to be $\geq_{D}$ $\mathrm{MOD}(\mathrm{W})$ and $\mathrm{AE}$ extensions to be minimal. This groundedness filter is not applied in circumscription. This more or less explains why we need a stronger version of preferential consequence for default logic than for circumscription.

\section{Acknowledgments}

I would like to thank Albert Visser for commenting on preliminary versions of this paper and the members of the (Dutch) National Working Group on Non-Monotonc Reasoning for enduring a presentation of one of those versions.

\section{References}

BLAMEY, S., 'Partial Logic', Handbook of Philosophical Logic Vol III, D. Gabbay and F. Guenthner (eds.), D. Reidel, Dordrecht, (1986) 1-70.

ETHERINGTON, D., Reasoning with Incomplete Information, Research Notes in Artificial Intelligence, Pitman, London (1988).

LANGHOLM, T., Partiality, Truth and Persistence, CSLI Lecture Notes 15, CSLI, Stanford (1986).

LUKASZEWICZ, L., Non-monotonic Reasoning: Formalization of Commonsense Reasoning, Ellis Horwood, Chichester (1990).

MAKINSON, D., 'General Theory of Cumulative Inference', Non-Monotonic Reasoning (Proceedings 2 nd Internatonal Workshop, Grassau, FRG, June 1988), M. Reinfrank, J. de Kleer, M. Ginsberg, E. Sandewall (eds.), Springer, Berlin (1989) 1-18.

MCCARTHY, J., 'Circumscription - a Form of Nonmonotonic Reasoning', Artificial Intelligence 13 (1980) 27-39.

REITER, R., 'A Logic for Default Reasoning', Artificial Intelligence 13 (1980) 81-132.

SHOHAM, Y., 'A Semantical Approach to Nonmonotonic Logics', Readings in Nonmonotonic Reasoning, M. Ginsberg (ed.), Morgan Kaufmann, Los Altos CA. (1987) 227-250.

SHOHAM, Y., Reasoning about Change, MIT Press, Cambridge MA, (1988).

VOORBRAAK, F., 'Epistemic Logic and Nonmonotonic Reasoning', forthcoming. 


\section{Logic Group Preprint Series \\ Department of Philosophy, University of Utrecht \\ Heidelberglaan 8, 3584 CS Utrecht \\ The Netherlands}

1 C.P.J. Koymans, J.L.M. Vrancken, Extending Process Algebra with the empty process, September 1985

2 J.A. Bergstra, A process creation mechanism in Process Algebra, September 1985

3 J.A. Bergstra, Put and get, primitives for synchronous unreliable message passing, October 1985

4 A. Visser, Evaluation, provably deductive equivalence in Heyting's arithmetic of substitution instances of propositional formulas, November 1985

5 G.R. Renardel de Lavalette, Interpolation in a fragment of intuitionistic propositional logic, January 1986

6 C.P.J. Koymans, J.C. Mulder, A modular approach to protocol verification using Process Algebra, April 1986

7 D. van Dalen, F.J. de Vries, Intuitionistic free abelian groups, April 1986

8 F. Voorbraak, A simplification of the completeness proofs for Guaspari and Solovay's $R$, May 1986

9 H.B.M. Jonkers, C.P.J. Koymans \& G.R. Renardel de Lavalette, A semantic framework for the COLDfamily of languages, May 1986

10 G.R. Renardel de Lavalette, Strictheidsanalyse, May 1986

11 A. Visser, Kunnen wij elke machine verslaan? Beschouwingen rondom Lucas' argument, July 1986

12 E.C.W. Krabbe, Naess's dichotomy of tenability and relevance, June 1986

13 H. van Ditmarsch, Abstractie in wiskunde, expertsystemen en argumentatie, Augustus 1986

14 A. Visser, Peano's Smart Children, a provability logical study of systems with built-in consistency, October 1986

15 G.R. Renardel de Lavalette, Interpolation in natural fragments of intuitionistic propositional logic, October 1986

16 J.A. Bergstra, Module Algebra for relational specifications, November 1986

17 F.P.J.M. Voorbraak, Tensed Intuitionistic Logic, January 1987

18 J.A. Bergstra, J. Tiuryn, Process Algebra semantics for queues, January 1987

19 F.J. de Vries, A functional program for the fast Fourier transform, March 1987

20 A. Visser, A course in bimodal provability logic, May 1987

21 F.P.J.M. Voorbraak, The logic of actual obligation, an alternative approach to deontic logic, May 1987

22 E.C.W. Krabbe, Creative reasoning in formal discussion, June 1987

23 F.J. de Vries, A functional program for Gaussian elimination, September 1987

24 G.R. Renardel de Lavalette, Interpolation in fragments of intuitionistic propositional logic, October 1987 (revised version of no. 15)

25 F.J. de Vries, Applications of constructive logic to sheaf constructions in toposes, October 1987

26 F.P.J.M. Voorbraak, Redeneren met onzekerheid in expertsystemen, November 1987

27 P.H. Rodenburg, D.J. Hoekzema, Specification of the fast Fourier transform algorithm as a term rewriting system, December 1987

28 D. van Dalen, The war of the frogs and the mice, or the crisis of the Mathematische Annalen, December 1987

29 A. Visser, Preliminary Notes on Interpretability Logic, January 1988

30 D.J. Hoekzema, P.H. Rodenburg, Gauß elimination as a term rewriting system, January 1988

31 C. Smoryński, Hilbert's Programme, January 1988

32 G.R. Renardel de Lavalette, Modularisation, Parameterisation, Interpolation, January 1988

33 G.R. Renardel de Lavalette, Strictness analysis for POLYREC, a language with polymorphic and recursive types, March 1988

34 A. Visser, A Descending Hierarchy of Reflection Principles, April 1988

35 F.P.J.M. Voorbraak, A computationally efficient approximation of Dempster-Shafer theory, April 1988

36 C. Smoryński, Arithmetic Analogues of McAloon's Unique Rosser Sentences, April 1988 